\title{
Efectos adversos a largo plazo de los inhibidores de la bomba de protones. Perspectiva desde la medicina basada en la evidencia
}

\section{Evidence Based Medicine Perspective on Long-Term Adverse Effects of Proton Pump Inhibitors}

Jaime A. Cardona-Ospina, MD, ${ }^{1,2}$ Diego Alejandro Medina-Morales, MD, ${ }^{1}$ Alfonso J. Rodríguez-Morales, MD, MSc, ${ }^{2}$

Jorge E. Machado-Alba, MD, MSc, PhD. ${ }^{3}$

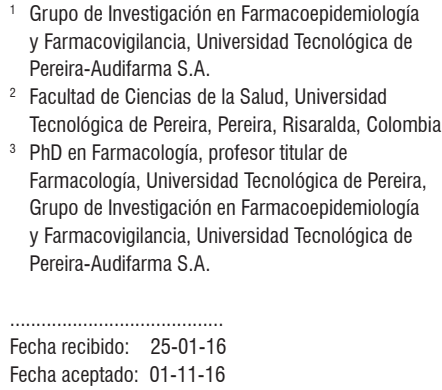
y Farmacovigilancia, Universidad Tecnológica de Pereira-Audifarma S.A.

2 Facultad de Ciencias de la Salud, Universidad Tecnológica de Pereira, Pereira, Risaralda, Colombia.

${ }^{3} \mathrm{PhD}$ en Farmacología, profesor titular de Farmacología, Universidad Tecnológica de Pereira, Grupo de Investigación en Farmacoepidemiología y Farmacovigilancia, Universidad Tecnológica de Pereira-Audifarma S.A.

Fecha recibido: $25-01-16$ Fecha aceptado: $01-11-16$

\begin{abstract}
Resumen
Los inhibidores de la bomba de protones (IBP) son los supresores de la secreción gástrica más efectivos y se encuentran entre los medicamentos de mayor formulación y venta en Estados Unidos; en algunos casos son prescritos sin indicación justificada. En años recientes, el reporte de reacciones adversas importantes relacionadas con su uso ha suscitado preocupación. Sin embargo, la calidad de la evidencia no ha sido concluyente y, en algunos casos, la magnitud del riesgo no es de importancia clínica. El objetivo de esta revisión es presentar la evidencia disponible frente a los eventos adversos de mayor importancia relacionados con los IBP.
\end{abstract}

\section{Palabras clave}

Inhibidores de la bomba de protones, efectos colaterales y reacciones adversas relacionadas con medicamentos, medicina basada en evidencia, farmacovigilancia (fuente: DeCS).

\begin{abstract}
Proton pump inhibitors (PPIs) are the most effective gastric secretion suppressors and are among the most widely prescribed and widely available drugs in the United States of America. In some cases they are prescribed without justification. In recent years, concerns have arisen over reports of major adverse reactions related to the use of PPIs. However, the quality of the evidence has not been conclusive, and in some cases the magnitude of the risk has not been clinically significant. The objective of this review is to present the available evidence regarding the most important adverse events related to PPIs.
\end{abstract}

Keywords

Proton pump inhibitors, adverse drug side effects, adverse drug reactions, evidence-based medicine, pharmacovigilance (Source: DeCS).

\section{INTRODUCCIÓN}

Los inhibidores de la bomba de protones (IBP) son medicamentos supresores de la secreción gástrica que actúan mediante la inhibición de $\mathrm{H}^{+} \mathrm{K}^{+}$-ATPasa en las células parietales del cuerpo y el fondo gástrico. Son profármacos que al ser ingeridos pasan a la circulación general y se difunden por las membranas de las células parietales hasta los cana- lículos, en donde el pH ácido (alrededor de 0,8) induce la formación de una sulfonamida tetracíclica que se une covalentemente con los grupos sulfidrilo de los aminoácidos cisteína en la $\mathrm{H}^{+} \mathrm{K}^{+}$-ATPasa inactivándola irreversiblemente e inhibiendo la secreción ácida gástrica hasta que nuevas bombas de protones son sintetizadas. Actualmente hay disponibles cinco IBP en el mercado: omeprazol, esomeprazol, lansoprazol, pantoprazol y rabeprazol, los cuales 
tienen diferencias en sus grupos piridina o benzimidazol, pero son similares en sus propiedades farmacológicas.

Estos medicamentos se encuentran entre los de mayor prescripción en Estados Unidos y la disposición de genéricos de bajo costo y su venta sin fórmula médica permite que los pacientes puedan disponer de ellos ampliamente (1). La necesidad de prescribirlos para tratamientos a largo plazo, los reportes de reacciones adversas relacionadas con medicamentos (RAM) de diferente índole, su sobreutilización en atención primaria, en los servicios de urgencias y hospitalización, y su prescripción sin indicación $(2,3)$, han propiciado la reflexión sobre su uso seguro y han impulsado la publicación de varios trabajos al respecto. Este trabajo revisa la literatura actual disponible frente a las RAM de mayor relevancia clínica.

\section{REACCIONES ADVERSAS ASOCIADAS CON IBP}

Las RAM asociadas con el empleo de IBP pueden ser clasificadas en dos grupos: a corto plazo o primarias y a largo plazo o secundarias. Del 1\% al 5\% de los pacientes reportan reacciones adversas primarias (cefalea, diarrea, estreñimiento, náuseas y rash), las cuales son comunes al grupo farmacológico y cuyas frecuencias no han sido comparadas entre IBP (4). Por otro lado, las RAM reportadas con su uso prolongado han sido sujeto de mayores estudios por su importancia clínica (tabla 1 ).

Tabla 1. Reacciones adversas secundarias asociadas con el uso de inhibidores de la bomba de protones.

\begin{tabular}{l} 
Tipo de reacción adversa a largo plazo \\
\hline Reducción del efecto antitrombótico del clopidogrel \\
Incremento del riesgo de osteoporosis y fracturas \\
Incremento del riesgo de neumonía adquirida en la comunidad y \\
neumonía nosocomial \\
Incremento del riesgo de infecciones por $C$. difficile y otras infecciones \\
entéricas \\
Eventos perinatales y postnatales adversos con el uso durante el \\
embarazo \\
Nefritis intersticial aguda \\
Deficiencia de vitamina $B_{12}$ \\
Hipomagnesemia \\
Inhibición de la absorción de hierro \\
Incremento en el riesgo de neoplasias \\
Aumento en el riesgo de peritonitis bacteriana espontánea en \\
pacientes con cirrosis \\
Neutropenia
\end{tabular}

\section{Reducción del efecto antitrombótico del clopidogrel}

Basados en datos farmacocinéticos, una interacción potencial entre el clopidogrel y el omeprazol produjo preocupación, dado que el primero requiere ser activado por la isoenzima CYP2C19 y el segundo la inhibe, mientras que el pantoprazol y el esomeprazol utilizan vías alternas. Experimentos in vitro reportaron una reducción en la acción biológica del clopidogrel sobre la función plaquetaria, pero estudios posteriores arrojaron respuestas contradictorias sobre la relevancia clínica de la interacción o la variación de resultados entre moléculas de IBP (5). El estudio COGENT (2010) concluyó que el uso de omeprazol con clopidogrel reduce el riesgo de eventos gastrointestinales, comparado con el uso de clopidogrel más placebo, sin incremento en el riesgo de eventos cardiovasculares (muerte, infarto agudo de miocardio no fatal, revascularización o accidente cerebrovascular). Sin embargo, este estudio fue suspendido tempranamente por pérdida del patrocinio (6). Incluyó 3627 de 5000 pacientes con un promedio de seguimiento de 133 días. Observó 136 eventos cardiovasculares (omeprazol: 69, placebo: 67; $\mathrm{p}=0,050$ ) y 105 eventos gastrointestinales (omeprazol: 38, placebo 67; $p=0,007$ ), $\mathrm{y}$ aunque no hubo diferencia significativa entre los eventos cardiovasculares de ambos grupos, los resultados no permitieron descartar una asociación clínicamente importante por su uso simultáneo. También, un subanálisis posterior, de los pacientes de alto riesgo cardiovascular incluidos en este estudio con tratamiento concomitante con omeprazol, encontró una reducción significativa de eventos gastrointestinales sin incremento del riesgo cardiovascular (7).

Por otro lado, un metaanálisis del 2012 que incluyó 27 investigaciones, con 159.998 pacientes, concluyó que el uso conjunto de IBP y clopidogrel se asociaba con un incremento del riesgo de eventos cardiovasculares mayores y a síndrome coronario agudo (HR: 1,40; IC95\%: 1,19-1,64; HR: 1,42; IC95\%: 1,14-1,77; respectivamente), pero no con mortalidad por cualquier causa, muerte cardiovascular o trombosis del stent (HR: 1,30; IC95\%: 0,91-1,91-1,86; HR: 1,21; IC95\%: 0,60-2,43, HR: 1,52; IC95\%: 0,87-2,65, respectivamente) (8). Sin embargo, durante el análisis individual solo el pantoprazol se asoció con incremento de riesgo de eventos cardiovasculares mayores (HR: 1,52; IC95\%: 1,18-1,94). Otro metaanálisis de 23 estudios con 222.311 participantes encontró que el riesgo cardiovascular era significativamente elevado al usar omeprazol, esomeprazol, lansoprazol más clopidogrel (OR: 1,24; IC95\%: 1,071,43; OR: 1,32; IC95\%: 1,09-1,60; OR: 1,41; IC95\%: 1,21$1,64)$. No obstante, en este mismo trabajo, un análisis de siete estudios observacionales halló incremento del riesgo cardiovascular con IBP sin clopidogrel (OR: 1,28; IC95\%: $1,14-1,44)$ versus no administrar ningún medicamento (9). 
En conclusión, hay un posible incremento en la probabilidad de eventos cardiovasculares mayores, pero no de desenlace fatal. Sin embargo, la heterogeneidad de los resultados y los datos que vinculan el aumento del riesgo cardiovascular en pacientes que reciben IBP sin clopidogrel sugieren un factor de confusión residual o la presencia de un mecanismo alterno que explique dicho efecto.

\section{Riesgo de osteoporosis y fracturas}

La absorción de calcio en el intestino delgado es facilitada por la ionización de sales insolubles a pH bajo. Inicialmente se postuló que el aumento del $\mathrm{pH}$ gástrico produciría una respuesta fisiológica compensatoria con incremento de la resorción ósea, secundaria a la elevación de la secreción de hormona paratiroidea, lo que produce un aumento del riesgo de osteoporosis y fracturas. Sin embargo, dada la presencia de una bomba de protones en osteoclastos, era también posible una reducción de la resorción ósea por el efecto inhibitorio del medicamento.

En 2010, se realizó un estudio de corte transversal que usó The Manitoba Bone Mineral Density Database con el fin de establecer la relación entre el uso crónico de IBP y osteoporosis o la pérdida acelerada de densidad ósea, comparándolas en el tiempo entre quienes usaban y no usaban IBP. Múltiples estudios coinciden en mostrar un escaso incremento en el riesgo de fracturas en general, con un aparente menor efecto en el riesgo de fracturas de columna, pero con heterogeneidad importante en sus resultados, y también una reducción en la mineralización del fémur con incremento del riesgo de osteopenia y osteoporosis (10, $11)$. Se encontró además que para fractura de cadera (OR: 1,25; IC95\%: 1,14-1,37), al ajustar por tiempo de uso, en los usuarios de IBP a corto plazo el incremento de riesgo era estadísticamente significativo (OR: 1,24; IC95\%: 1,191,28 ), al contrario que en los usuarios a largo plazo (OR: 1,30; IC95\%: 0,98-1,70) (12). Aunque la asociación es probable, la heterogeneidad de los datos no permite formular una conclusión definitiva.

\section{Incremento del riesgo de neumonía adquirida en la comunidad (NAC) y neumonía nosocomial (NNS)}

El pH gástrico ácido funciona como una barrera que impide el crecimiento de microorganismos en el tracto gastrointestinal. Múltiples estudios han documentado un mayor crecimiento bacteriano con el uso de IBP, el cual por lo general carece de importancia clínica (13). Sin embargo, debido a eventuales episodios de microaspiración secundarios a reflujo gastroesofágico, alteración del $\mathrm{pH}$ de las secreciones pulmonares o alteración de la función fagocítica, se ha generado una preocupación en torno a un incremento en el riesgo de NAC y NNS (14).

Con respecto al riesgo de NAC, los estudios observacionales parecen sustentar la asociación especialmente con el uso por menos de 30 días y a dosis altas. En 2012, un metaanálisis de nueve estudios de cohorte y casos y controles analizó el riesgo de NAC basado en el uso actual de IBP (OR: 1,39, IC95\%: 1,09-1,76), uso por menos de 30 días (OR: 1,65; IC95\%: 1,25-2,19), por más de 180 días (OR: 1,10; IC95\%: 1,00-1,21), a dosis bajas (OR: 1,17; IC95\%: 1,11-1,24) y dosis altas (OR: 1,50; IC95\%: 1,33-1,68) (15).

En lo referente a la NNS, los resultados apoyan consistentemente la asociación. En 2011, un metaanálisis mostró incremento del riesgo con la administración de IBP (RR: 1,22; IC95\%: 1,01-1,48, I ${ }^{(2)} 30,6 \%$ ), sin diferencia al comparar anti- $\mathrm{H}_{2}$ e IBP (16), siendo los IBP más efectivos para la profilaxis de úlceras de estrés con una tasa significativamente más baja de sangrado gastrointestinal (17). La evidencia respalda la asociación del uso de IBP con un incremento del riesgo de NAC y NNS, pero el mecanismo fisiopatológico por el cual esto ocurre aún no ha sido dilucidado.

\section{Riesgo de infecciones por $C$. difficile y otras infecciones entéricas}

Además del mayor crecimiento bacteriano intestinal, los IBP tienen efectos inmunomoduladores y antiinflamatorios, por lo que se ha sugerido que favorecen la traslocación bacteriana. Y dado que algunos microorganismos muestran diferente tolerancia a distintos niveles de $\mathrm{pH}$ gástrico pudiera incrementarse el riesgo de infección por determinados patógenos, especialmente: Salmonella spp., Campylobacter jejuni, Escherichia coli enteroinvasiva, Clostridium difficile, Vibrio cholerae y Listeria spp. (18). Varios metaanálisis han hallado un incremento en el riesgo de infecciones, especialmente por C. difficile, aunque ha habido heterogeneidad entre los resultados e interacción entre variables, en particular por el uso concomitante de antibióticos.

Kwok y colaboradores, en 2012, encontraron para el riesgo de infección por C. difficile un OR de 1,74 (IC95\%: $\left.1,47-2,85, \mathrm{I}^{(2)}=78 \% ; \mathrm{p}=0,005\right)$ con mayor riesgo si conjuntamente recibían tratamiento antibiótico (OR: 1,96; IC95\%: 1,03-3,70); el índice de sinergia de Rothman fue de 1,36 , con una proporción de riesgo atribuible a la interacción de 0,19 , lo que indica un riesgo incrementado más allá del efecto de cada medicamento aislado (19). Sin embargo, Tleyjeh y colaboradores, en el año 2012, concluyeron que se cuenta con evidencia de muy baja calidad y por ello no se puede establecer una relación causal, lo que sugiere que, en caso de incrementarse el riesgo de infección por C. difficile, el NNH sería de 3.925 pacientes en un año (20). 


\section{Riesgo de eventos perinatales y postnatales adversos}

Se han realizado múltiples estudios de cohorte y metaanálisis para buscar la asociación entre el uso de IBP y malformaciones congénitas $u$ otros eventos perinatales adversos (21) y los resultados sugieren su seguridad, especialmente para omeprazol, incluso cuando se administra durante el primer trimestre de la gestación. Sin embargo, datos recientes muestran un incremento en el riesgo de asma infantil en hijos de madres expuestas al uso de cualquier tipo de antiulceroso (OR: 1,23; IC95\%: 1,01-1,51) (22).

\section{Riesgo de lesión renal aguda}

Se han publicado estudios que asocian el uso de IBP con lesión renal aguda y nefritis intersticial. Klepser y colaboradores encontraron una asociación entre enfermedad renal y uso de IBP (OR: 1,72; IC95\%: 1,27-2,32), la cual persistía incluso después de hacer ajustes por variables confusoras (23). Sin embargo, dada la naturaleza de los estudios y el número de eventos reportados, no pueden formularse conclusiones definitivas al respecto.

\section{Deficiencia de vitamina $B_{12}$}

La cianocobalamina se obtiene de una dieta rica en proteínas que deben ser escindidas por proteasas pancreáticas activadas por la secreción ácida gástrica, que luego debe ser absorbida en el íleon terminal tras unirse al factor intrínseco. En el 2008 se publicó un estudio transversal en el que se compararon 125 pacientes mayores de 65 años, usuarios de IBP por más de 3 años, frente a sus parejas no consumidoras del medicamento, y no se encontraron diferencias en los niveles de vitamina $B_{12}(p=0,730)$, incluso después de ajustar por la presencia o no de Helicobacter pylori, edad, sexo y niveles de proteína $\mathrm{C}$ reactiva. Además, tampoco se encontraron diferencias en los niveles de homocisteína ni en los valores de volumen corpuscular medio (24).

Más recientemente, se analizaron 36 pacientes de entre 60 y 80 años, internados ( 17 en terapia a largo plazo, 19 no usuarios de IBP), en los que se valoraron los niveles basales de vitamina $B_{12}$ y el efecto de su administración en aerosol nasal durante 8 semanas. Se encontró que los usuarios crónicos de IBP tenían niveles basales más bajos de esta vitamina y niveles más altos de ácido metilmalónico (75\% vs. $11 \% ; \mathrm{p}=0,006)(25)$. También se ha encontrado asociación entre tratamiento concomitante con metformina, IBP o Anti-H2 y deficiencia de vitamina $B_{12}$ (26). Por el escaso tamaño de las muestras y el diseño de los estudios es difícil hacer conclusiones definitivas, si bien debe considerarse la mayor posibilidad de riesgo de déficit de vitamina $B_{12}$, especialmente en ancianos con terapia crónica, mal estado nutricional o pacientes con diabetes mellitus y tratamiento concomitante con metformina.

\section{Riesgo de hipomagnesemia}

En 2006 se reportó la asociación de hipomagnesemia e IBP. Desde ese entonces se han realizado al menos 13 reportes de alteraciones hidroelectrolíticas asociadas (hipomagnesemia, hipocalcemia, hipocalemia e hipofosfatemia) (27). Las manifestaciones clínicas descritas incluyen desde síntomas gastrointestinales (náuseas, vómito, diarrea), síntomas musculo-esqueléticos (calambres y temblores), hasta arritmias, convulsiones y edema pulmonar (28-30). Aparentemente el proceso fisiopatológico por el cual se produce la hipomagnesemia guarda relación con la alteración de la absorción de este ion a nivel gastrointestinal y corresponde a un efecto de clase (30). Es probable que la duración del tratamiento sea un factor de riesgo pues la mayoría de casos reportados llevan más de un año de uso, aunque también se ha reportado con tratamientos de tres meses (30). La FDA recomienda vigilar el magnesio sérico antes del inicio de terapia a largo plazo (mayor a 1 año) o la medicación simultánea con otros medicamentos relacionados con hipomagnesemia (diuréticos, digoxina, entre otros) (31).

\section{Inhibición de la absorción de hierro}

El hierro de la dieta está presente en dos formas: ferroso, de fácil absorción, y férrico, el cual debe ser transformado a la forma ferrosa en una reacción dependiente del $\mathrm{pH}$ ácido. La evidencia actual sobre la relación entre IBP y déficit de hierro es sustentada por casos de pacientes con anemia ferropénica quienes no respondieron a los suplementos sino hasta que se suspendieron los IBP. Además, ha habido reportes de cambios hematológicos que indican deficiencia de hierro en pacientes con IBP por más de 1 año, y de pacientes con hemocromatosis hereditaria quienes tras el uso de IBP a largo plazo mostraron una reducción significativa en los requerimientos de flebotomía (32). No obstante, en pacientes que consumieron omeprazol por más de 4 años, y otros con síndrome de Zollinger-Ellison en tratamiento con IBP por 6 años, no se encontró disminución de los depósitos de hierro corporales (33). Los resultados actuales sugieren que la relación es teóricamente posible, pero no han sido demostrados clínicamente y es improbable que sea de importancia en pacientes con reservas normales de hierro (32).

\section{Riesgo de neoplasias gastrointestinales}

El tratamiento prolongado con IBP eleva el $\mathrm{pH}$ gástrico, lo que incrementa la secreción de gastrina y sus niveles plasmáticos. Los efectos tróficos de esta hormona llevan a 
hiperplasia de células similares a enterocromafines, por lo que se ha planteado que el tratamiento con IBP conduciría al desarrollo de pólipos gástricos, cáncer gástrico, cáncer colorrectal y tumores carcinoides (33). Algunos datos sugieren un aumento del riesgo de gastritis atrófica, especialmente en personas con $H$. pylori; sin embargo, no hay evidencia suficiente que apoye su progresión a enfermedad maligna (33). Un metaanálisis de 2013, de seis ensayos clínicos aleatorios, evaluó el riesgo de lesiones premalignas o malignas con el uso de IBP por más de 6 meses, pero no encontró asociación, incluso en pacientes con enfermedad por reflujo gastroesofágico y terapia de mantenimiento (34).

Respecto al riesgo de cáncer de colorrectal, un estudio con seguimiento de 15 años mostró que la terapia con IBP a largo plazo, a dosis regulares, no se asocia con aumento significativo del riesgo de dicho cáncer (35). Así, en la actualidad no hay evidencia clínica de incremento del riesgo de lesiones malignas o premalignas a nivel gástrico o colónico. No hay estudios epidemiológicos que hayan evaluado la asociación entre el tratamiento con IBP y el riesgo de cáncer de esófago, páncreas y tumores carcinoides.

\section{Peritonitis bacteriana espontánea en pacientes con cirrosis}

Se ha sugerido que los IBP pueden aumentar el riesgo de infecciones en pacientes con cirrosis y predisponer a peritonitis bacteriana espontánea (PBE), posiblemente por aumento de la permeabilidad intestinal y el mayor crecimiento bacteriano (36). La evidencia al respecto se fundamenta en estudios retrospectivos de pacientes cirróticos hospitalizados con ascitis, en los que el uso reciente de IBP se asoció en forma independiente a PBE, pero sin alterar la sobrevida a un mes, tres meses o un año (36). Adicionalmente, Goel y colaboradores, en 2012, encontraron que los pacientes con PBE tienen una incidencia significativamente más alta tras uso reciente (en los pasados siete días) de IBP $(71,0 \%)$ que los pacientes control $(42,0 \%)$. Sin embargo, también mostraron que no hay diferencia entre los pacientes que recibieron IBP entres los $8 y$ 90 días previos versus aquellos quienes no los usaron $(\mathrm{p}=$ 0,58 ) (37). Kwon y colaboradores, en 2014, hallaron que tanto la terapia con IBP como con antagonistas $\mathrm{H}_{2}$ estuvo asociada con PBE, y que el uso de IBP en los 30 días previos se asoció con mayor mortalidad, independiente de la severidad de la enfermedad hepática de base (OR: 1,96; IC95\%: 1,19-3,22; $p=0,008$ ) (38). Por el contrario, Bajaj y colaboradores, en 2012, no hallaron asociación entre el uso de IBP y la tasa de infecciones serias (HR: 1,08; IC95\%: $0,90-1,31)$ (39). De acuerdo con lo anterior, y por la falta de información concluyente, no se pueden plantear conclusiones al respecto.

\section{Leucopenia, neutropenia y agranulocitosis}

La leucopenia, la neutropenia o la agranulocitosis asociadas con IBP son efectos indeseables poco descritos en la literatura. Se plantea que pueden ser explicados por reacción inmunológica o por toxicidad directa, y se ha sugerido que pueden ser un efecto de clase. Sin embargo, otros supuestos sugieren una diferencia en el mecanismo de acuerdo con el IBP considerado (40). Se precisan más reportes para aclarar la relación causa-efecto de esta asociación.

\section{CONCLUSIÓN}

Aunque se ha sugerido la asociación entre el consumo de IBP y diversas RAM a largo plazo, la calidad de la evidencia para sustentar el incremento del riesgo para cada una de ellas es heterogénea y en muchos casos de calidad marginal. No obstante, existen asociaciones fuertes e importantes, como el incremento del riesgo de osteopenia, osteoporosis, fracturas o de neumonía nosocomial, que, dado su impacto en desenlaces a largo plazo, deben alertar al clínico sobre la necesidad de balancear el riesgo, costo y beneficio de su utilización.

Se debe tener presente que la utilización indiscriminada, y ante todo sin indicación, de medidas terapéuticas incrementa el riesgo de eventos indeseados y la aparición incluso de reacciones infrecuentes. Por ello, se debe garantizar la administración de IBP solo en aquellos pacientes con una indicación clara y apoyada en la evidencia, y la suspensión del medicamento en los casos en que sea necesario hacerlo.

\section{REFERENCIAS}

1. Vakil N. Prescribing proton pump inhibitors: is it time to pause and rethink? Drugs. 2012; 72(4):437-45.

2. Batuwitage BT, Kingham JG, Morgan NE, et al. Inappropriate prescribing of proton pump inhibitors in primary care. Postgrad Med J 2007; 83(975): 66-8.

3. Ramirez E, Lei SH, Borobia AM, et al. Overuse of PPIs in patients at admission, during treatment, and at discharge in a tertiary Spanish hospital. Curr Clin Pharmacol 2010; 5(4): 288-97.

4. Chubineh S, Birk J. Proton Pump Inhibitors: The Good, the Bad, and the Unwanted. South Med J. 2012; 105(11):613-8.

5. Gilard M, Arnaud B, Le Gal G, et al. Influence of omeprazol on the antiplatelet action of clopidogrel associated to aspirin. J Thromb Haemost. 2006; 4(11):2508-9.

6. Bhatt DL, Cryer BL, Contant CF, et al. Clopidogrel with or without omeprazole in coronary artery disease. N Engl J Med. 2010; 363(20):1909-17.

7. Vaduganathan M, Cannon CP, Cryer BL, Liu Y, Hsieh WH, Doros G, et al. Efficacy and Safety of Proton-Pump Inhibitors in High-Risk Cardiovascular Subsets of the COGENT Trial. Am J Med. 2016. 
8. Huang B, Huang Y, Li Y, et al Adverse cardiovascular effects of concomitant use of proton pump inhibitors and clopidogrel in patients with coronary artery disease: a systematic review and meta-analysis. Arch Med Res. 2012; 43(3):212-24.

9. Kwok CS, Jeevanantham V, Dawn B, et al. No consistent evidence of differential cardiovascular risk amongst protonpump inhibitors when used with clopidogrel: meta-analysis. Int J Cardiol. 2013; 167(3):965-74.

10. Ngamruengphong S, Leontiadis GI, Radhi S, et al. Proton pump inhibitors and risk of fracture: a systematic review and meta-analysis of observational studies. Am J Gastroenterol. 2011; 106(7):1209-18.

11. Arj A, Razavi Zade M, Yavari M, et al. Proton pump inhibitors use and change in bone mineral density. Int J Rheum Dis. 2016.

12. Kwok CS, Yeong JK, Loke YK. Meta-analysis: risk of fractures with acid-suppressing medication. Bone. 2011; 48(4):768-76.

13. Williams C, McColl KE. Review article: proton pump inhibitors and bacterial overgrowth. Aliment Pharmacol Ther. 2006; 23(1):3-10.

14. Proton pump inhibitors: bacterial pneumonia. Prescrire Int. 2012; 21(130):210-2.

15. Giuliano C, Wilhelm SM, Kale-Pradhan PB. Are proton pump inhibitors associated with the development of community-acquired pneumonia? A meta-analysis. Expert Rev Clin Pharmacol. 2012; 5(3):337-44

16. Eom CS, Jeon CY, Lim JW, et al. Use of acid-suppressive drugs and risk of pneumonia: a systematic review and metaanalysis. CMAJ. 2011; 183(3):310-9.

17. Lin PC, Chang CH, Hsu PI, et al. The efficacy and safety of proton pump inhibitors vs histamine- 2 receptor antagonists for stress ulcer bleeding prophylaxis among critical care patients: a meta-analysis. Crit Care Med 2010; 38(4):1197-205.

18. Bavishi C, Dupont HL. Systematic review: the use of proton pump inhibitors and increased susceptibility to enteric infection. Aliment Pharmacol Ther 2011; 34(11-12):1269-81.

19. Kwok CS, Arthur AK, Anibueze CI, et al. Risk of Clostridium difficile infection with acid suppressing drugs and antibiotics: meta-analysis. Am J Gastroenterol 2012; 107(7):1011-9.

20. Tleyjeh IM, Bin Abdulhak AA, Riaz M, et al. Association between proton pump inhibitor therapy and clostridium difficile infection: a contemporary systematic review and meta-analysis. PLoS One.2012; 7(12):e50836.

21. Gill SK, O'Brien L, Einarson TR, et al. The safety of proton pump inhibitors in pregnancy: a meta-analysis. Am J Gastroenterol. 2009; 104(6):1541-5.

22. Hak E, Mulder B, Schuiling-Veninga CC, et al. Use of AcidSuppressive Drugs in Pregnancy and the Risk of Childhood Asthma: Bidirectional Crossover Study using the General Practice Research Database. Drug Saf. 2013; 36(11):1097-104.

23. Klepser DG, Collier DS, Cochran GL. Proton pump inhibitors and acute kidney injury: a nested case-control study. BMC Nephrol. 2013; 14(1):150.

24. den Elzen WP, Groeneveld Y, de Ruijter W, et al. Longterm use of proton pump inhibitors and vitamin B12 sta- tus in elderly individuals. Aliment Pharmacol Ther. 2008; 27(6):491-7.

25. Rozgony NR, Fang C, Kuczmarski MF, et al. Vitamin B(12) deficiency is linked with long-term use of proton pump inhibitors in institutionalized older adults: could a cyanocobalamin nasal spray be beneficial? J Nutr Elder. 2010; 29(1):87-99.

26. Damiao CP, Rodrigues AO, Pinheiro MF, et al. Prevalence of vitamin B12 deficiency in type 2 diabetic patients using metformin: a cross-sectional study. Sao Paulo Med J. 2016.

27. Epstein M, McGrath S, Law F. Proton-pump inhibitors and hypomagnesemic hypoparathyroidism. N Engl J Med. 2006; 355(17):1834-6.

28. Mackay JD, Bladon PT. Hypomagnesaemia due to protonpump inhibitor therapy: a clinical case series. QJM. 2010; 103(6):387-95.

29. Hoorn EJ, van der Hoek J, de Man RA, et al. A case series of proton pump inhibitor-induced hypomagnesemia. Am J Kidney Dis. 2010; 56(1):112-6.

30. Florentin M, Elisaf MS. Proton pump inhibitor-induced hypomagnesemia: A new challenge. World J Nephrol. 2012; 1(6):151-4.

31. U.S. Food and Drug Administration. FDA Drug Safety Communication: Low magnesium levels can be associated with long-term use of Proton Pump Inhibitor drugs (PPIs) http: / / www.fda.gov/drugs/drugsafety/ucm245011. htm2011 [cited 2014 02/09].

32. Reimer C. Safety of long-term PPI therapy. Best Pract Res Clin Gastroenterol. 2013; 27(3):443-54

33. Yang YX, Metz DC. Safety of proton pump inhibitor exposure. Gastroenterology. 2010; 139(4):1115-27.

34. Eslami L, Nasseri-Moghaddam S. Meta-analyses: does long-term PPI use increase the risk of gastric premalignant lesions? Arch Iran Med. 2013; 16(8):449-58.

35. Robertson DJ, Larsson H, Friis $S$, et al. Proton pump inhibitor use and risk of colorectal cancer: a population-based, case-control study. Gastroenterology. 2007; 133(3):755-60.

36. Trikudanathan G, Israel J, Cappa J, et al. Association between proton pump inhibitors and spontaneous bacterial peritonitis in cirrhotic patients - a systematic review and meta-analysis. Int J Clin Pract. 2011; 65(6):674-8.

37. Goel GA, Deshpande A, Lopez R, et al. Increased rate of spontaneous bacterial peritonitis among cirrhotic patients receiving pharmacologic acid suppression. Clin Gastroenterol Hepatol. 2012; 10(4):422-7.

38. Kwon JH, Koh SJ, Kim W, et al. Mortality associated with proton pump inhibitors in cirrhotic patients with spontaneous bacterial peritonitis. J Gastroenterol Hepatol. 2014; 29(4):775-81.

39. Bajaj JS, Ratliff SM, Heuman DM, et al. Proton pump inhibitors are associated with a high rate of serious infections in veterans with decompensated cirrhosis. Aliment Pharmacol Ther. 2012; 36(9):866-74.

40. Gouraud A, Vochelle V, Descotes J, et al. Proton pump inhibitor-induced neutropenia: possible cross-reactivity between omeprazole and pantoprazole. Clin Drug Investig. 2010; 30(8):559-63. 